\title{
Research
}

\section{Efficacy of increased resistant starch consumption in human type 2 diabetes}

\section{L Bodinham, L Smith, E L Thomas ${ }^{1}$, J D Bell ${ }^{1}$, J R Swann², A Costabile², D Russell-Jones, A M Umpleby and M D Robertson}

Nutrition, Metabolism and Diabetes Research Group, Faculty of Health and Medical Sciences, University of Surrey, Leggett Building, Guildford, Surrey GU2 7WG, UK

${ }^{1}$ Metabolic and Molecular Imaging Group, MRC Clinical Sciences Centre, Imperial College, London W12 0HS, UK and ${ }^{2}$ Department of Food and Nutritional Sciences, University of Reading, Whiteknights Campus, Reading RG6 6AP, UK
Correspondence should be addressed to M D Robertson

Email

m.robertson@surrey.ac.uk

\begin{abstract}
Resistant starch (RS) has been shown to beneficially affect insulin sensitivity in healthy individuals and those with metabolic syndrome, but its effects on human type 2 diabetes (T2DM) are unknown. This study aimed to determine the effects of increased RS consumption on insulin sensitivity and glucose control and changes in postprandial metabolites and body fat in T2DM. Seventeen individuals with well-controlled T2DM (HbA1c $46.6 \pm 2 \mathrm{mmol} / \mathrm{mol}$ ) consumed, in a random order, either $40 \mathrm{~g}$ of type $2 \mathrm{RS}$ (HAM-RS2) or a placebo, daily for 12 weeks with a 12-week washout period in between. At the end of each intervention period, participants attended for three metabolic investigations: a two-step euglycemic-hyperinsulinemic clamp combined with an infusion of $\left[6,6-{ }^{2} \mathrm{H}_{2}\right]$ glucose, a meal tolerance test (MTT) with arterio-venous sampling across the forearm, and whole-body imaging. HAM-RS2 resulted in significantly lower postprandial glucose concentrations $(P=0.045)$ and a trend for greater glucose uptake across the forearm muscle $(P=0.077)$; however, there was no effect of HAM-RS2 on hepatic or peripheral insulin sensitivity, or on HbA1c. Fasting non-esterified fatty acid (NEFA) concentrations were significantly lower $(P=0.004)$ and NEFA suppression was greater during the clamp with HAM-RS2 ( $P=0.001)$. Fasting triglyceride $(\mathrm{TG})$ concentrations and soleus intramuscular TG concentrations were significantly higher following the consumption of HAM-RS2 ( $P=0.039$ and $P=0.027$ respectively). Although fasting GLP1 concentrations were significantly lower following HAM-RS2 consumption ( $P=0.049)$, postprandial GLP1 excursions during the MTT were significantly greater $(P=0.009)$. HAM-RS2 did not improve tissue insulin sensitivity in well-controlled T2DM, but demonstrated beneficial effects on meal handling, possibly due to higher postprandial GLP1.
\end{abstract}

\author{
Key Words \\ - euglycemic-hyperinsulinemic \\ clamp \\ - GLP1 \\ - flux \\ stable isotopes
}

\section{Introduction}

It is estimated that 25.8 million children and adults in the USA have diabetes $(8.3 \%$ of the population), equating to a health cost of $\$ 218$ billion ( $\sim 10 \%$ of total healthcare expenditure). Lifestyle interventions, predominantly modulations to dietary intake, are the first-line strategy in diabetes treatment and remain a constant theme throughout management.

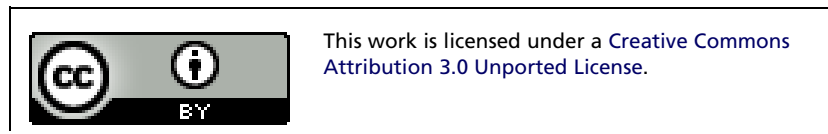


Traditionally, dietary fibers have been used to manage blood glucose concentration and have been linked to improved glycemic control in both healthy groups and those with diabetes through various meta-analyses (1). The USDA-recommended fiber intake is $14 \mathrm{~g} / 1000 \mathrm{kcal}$ in healthy individuals, with evidence currently lacking to recommend a higher intake in people with diabetes. This was highlighted in a scientific advisory committee (SACN) statement on nutrition that stated, although cereal fiber intake has been associated with a reduced incidence of type 2 diabetes (T2DM) and metabolic risk factors such as insulin resistance, the majority of evidence relates to T2DM prevention. While this is important given the current obesity epidemic, it cannot necessarily be translated into health benefits for those patients with T2DM (2).

Resistant starch (RS) is a type of cereal fiber and has been shown to have beneficial effects on insulin sensitivity and fatty acid (FA) metabolism in both healthy individuals and those with metabolic syndrome $(3,4,5,6,7)$. However, the efficacy of RS in individuals with T2DM has not been investigated.

Animal studies have consistently shown that RS improves glucose and insulin metabolism through increased postprandial GLP1 secretion due to stimulation of the colonic enteroendocrine cells $(8,9)$. This can result in improved insulin secretion. Most recently our own data has shown restored first-phase insulin secretion in metabolic syndrome (10); however, the lack of translational work has recently been highlighted (11). The ADA position on RS states 'there are no published long-term studies in subjects with diabetes to prove benefits from the use of resistant starch' (12), whereas the Diabetes Nutrition Study Group of the EASD (13) makes no mention of RS, stating that 'total dietary fiber should ideally be more than $20 \mathrm{~g} / 1000 \mathrm{kcal}^{\prime}$. The current USA and UK fiber intakes equate to $\sim 7 \mathrm{~g}$ and $6 \mathrm{~g} / 1000 \mathrm{kcal}$, respectively, falling short of even modest guideline daily amounts (GDA).

The primary aim of the study was to translate the beneficial effects of RS feeding reported in healthy and insulin resistant groups, into similar observations in a T2DM cohort. Using an integrated, whole-body approach, stable isotope tracers to differentiate between changes in hepatic and peripheral insulin sensitivity of glucose uptake, body composition analysis using magnetic resonance imaging (MRI) and magnetic resonance spectroscopy (MRS) to investigate changes in body fat depot distribution, and a meal tolerance test (MTT) combined with arterio-venous (A-V) sampling across muscle tissue, for the first time we investigated the efficacy of increasing
RS intake to achieve total fiber intakes above the GDA in individuals with T2DM.

\section{Subjects and methods}

\section{Patients}

Seventeen individuals with T2DM (12 males, five females; mean age 55 (s.E.M. 2.4) years, mean BMI 30.6 (s.E.M. 1.3) $\mathrm{kg} / \mathrm{m}^{2}$ ) were enrolled in this study. All participants had well-controlled diabetes (mean HbA1c levels of 46.6 (s.E.M. 2) $\mathrm{mmol} / \mathrm{mol}$ at screening) and were diet and exercise controlled (2/17), taking metformin (13/17) or metformin and pioglitazone (2/17), were weight stable, and excluded if they had a history of gastrointestinal, cardiovascular, or other endocrine diseases.

The study was conducted according to the guidelines laid down in the Declaration of Helsinki, and all procedures were approved by the Kent NHS Research Ethics Committee (10/H1101/29) and the University of Surrey Research Ethics Committee. Written informed consent was obtained from all patients. This trial was registered via the International Standard Randomised Controlled Trial Number 10727538.

\section{Study design}

The study was carried out as a single-blind, randomized dietary intervention crossover study, comparing RS derived from maize with a placebo that was matched for available carbohydrate content. Participants were supplied with supplements labeled either ' $A$ ' or ' $B$ ' and were not made aware of their composition. Each supplement was consumed daily for 12 weeks with a 12 -week washout period between interventions. During the last week of each intervention period, participants completed a 7-day food and drink diary and a 7-day bowel habit and symptom diary. Participants attended for three study visits at the end of each intervention: i) a two-step euglycemic-hyperinsulinemic clamp combined with an infusion of $\left[6,6-{ }^{2} \mathrm{H}_{2}\right]$ glucose, ii) a MTT with $\mathrm{A}-\mathrm{V}$ sampling across the forearm muscle, and iii) a MRI scan. The first two studies were conducted at the Royal Surrey County Hospital, UK and the third at the MRC Clinical Sciences Center, Hammersmith Hospital, UK. Before each study visit, patients consumed a standard evening meal and then fasted for $12 \mathrm{~h}$. They were instructed to avoid strenuous exercise and alcohol for the preceding $24 \mathrm{~h}$.

Participants were randomized to either $67 \mathrm{~g}$ Hi-maize 260 (comprising 60\% RS and 40\% rapidly digestible starch (RDS) providing $40 \mathrm{~g}$ type $2 \mathrm{RS}$ derived from maize,

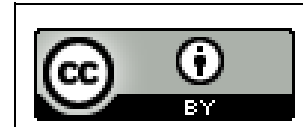

This work is licensed under a Creative Commons Attribution 3.0 Unported License. 
as measured by The Association of Official Analytical Chemists for total dietary fiber method 991.43) or $27 \mathrm{~g}$ Amioca (100\% RDS). Both supplements were supplied by Ingredion, Inc. (Bridgewater, NJ, USA) in ready-to-use sachets that were mixed into a beverage, and participants were required to consume two sachets daily to obtain the correct dosage. In the results and discussion, the Hi-maize supplement will be referred to as HAM-RS2 and the Amioca supplement as placebo.

\section{Euglycemic-hyperinsulinemic clamp with stable} isotopes - Participants arrived fasting and following voiding, body weight and composition were measured by bioimpedance (Tanita, Arlington Heights, IL, USA). An i.v. cannula was inserted into each arm for blood sampling and for the glucose, insulin, and isotope infusions. An initial blood sample was collected and a primed continuous infusion of $\left[6,6-{ }^{2} \mathrm{H}_{2}\right]$ glucose $(170 \mathrm{mg} ; 1.7 \mathrm{mg} / \mathrm{min})$ commenced. Once a steady state had been reached, five blood samples were taken between 100 and $120 \mathrm{~min}$. At $120 \mathrm{~min}$, the two-step euglycemic-hyperinsulinemic clamp was started. Step one assessed hepatic (endogenous) glucose production (EGP) and involved an insulin infusion (Actrapid, Novo Nordisk, Bagsvaerd, Denmark) of $0.3 \mathrm{mU} / \mathrm{kg}$ per min (low dose) for $120 \mathrm{~min}$. Step two assessed insulin sensitivity of glucose uptake (disposal, $\mathrm{Rd}$ ) and involved an insulin infusion of $1.5 \mathrm{mU} / \mathrm{kg}$ per min (high dose) for a further $180 \mathrm{~min}$. Plasma glucose concentrations were maintained at fasting levels through a variable infusion of $20 \%$ dextrose spiked with $\left[6,6-{ }^{2} \mathrm{H}_{2}\right]$ glucose $(8 \mathrm{mg} / \mathrm{g}$ for low dose and $10 \mathrm{mg} / \mathrm{g}$ for the high dose). Blood samples were taken every $10 \mathrm{~min}$ and blood glucose concentrations measured immediately by the glucose oxidase method using the YSI 2300 STAT Plus (YSI Life Sciences, Fleet, UK). Additional samples were taken during the steady state of the low (210-240 min) and high (390-420 min) dose steps.

Owing to difficulties with venous access in some individuals, the data for the euglycemic-hyperinsulinemic clamp are presented for $n=15$ only.

MTT with A-V sampling • Following a rest of $10 \mathrm{~min}$, carotid-femoral pulse wave velocity, brachial pulse wave analysis (including measures of mean arterial pressure, aortic pulse pressure, augmentation index, stroke volume, systolic and diastolic aortic blood pressures, and total peripheral resistance), and blood pressure were assessed using the Vicorder system (Smart Medical, Inc., La Mirada, CA, USA).

To assess the metabolism of skeletal muscle in vivo, the $\mathrm{A}-\mathrm{V}$ difference across the forearm muscle was assessed as described previously (5). Arterialized blood was obtained from a cannula inserted into the hand that was placed in a heated box $\left(50{ }^{\circ} \mathrm{C}\right)$. Venous blood was obtained from a cannula placed into deep muscle draining vein of the opposite arm and the hand was occluded for $2 \mathrm{~min}$ before drawing each sample using a wrist cuff inflated to $20 \mathrm{mmHg}$ above systolic blood pressure. Oxygen saturation was assessed at each site to ensure correct placement and arterialization, with cutoffs of $<60 \% \mathrm{O}_{2}$ for venous blood and $>95 \% \mathrm{O}_{2}$ for arterialized blood.

Two fasting blood samples were taken simultaneously from both sites. Patients then consumed a standardized liquid meal that did not contain either of the supplements (436 kcal, $61.2 \mathrm{~g}$ carbohydrate, $11.9 \mathrm{~g}$ fat, and $0 \mathrm{~g}$ dietary fiber) at time 0 . Further blood samples were collected simultaneously from both sites every half hour for $5 \mathrm{~h}$.

Owing to difficulties with venous access, the postprandial data presented are for 16 patients only and the A-V difference data are for 12 patients only.

Magnetic resonance imaging $>$ Upon arrival at the MRI unit, patients underwent whole-body MRI scanning to measure total and regional adipose tissue (AT) contents, as well as liver, pancreatic, and muscle proton MRS $\left({ }^{1} \mathrm{H}-\mathrm{MRS}\right)$ measurements as described previously (3). As not all individuals were suitable candidates for MRI scanning, the data presented are for 14 patients only.

\section{Biochemistry}

Metabolites from the placebo and HAM-RS2 arms of the trial were analyzed together with an intra-assay variation $<2.5 \%$ for all metabolites.

Plasma samples collected during the clamp were analyzed enzymatically for glucose concentration using a Cobas Mira (Roche Laboratories). Isotopic enrichment of the same plasma samples was measured by gas chromatography-mass spectrometry on an HP5971A mass selective detector (Agilent, Santa Clara, CA, USA). The enrichment was determined using a penta-O-trimethylsilyl-D-glucose$O$-methoxime derivative analyzed by selected ion monitoring of the ions at a charge-to-mass ratio of 319 and 321 (14). Plasma glucose concentrations from the MTT were measured using the glucose oxidase method on the YSI 2300 STAT Plus (YSI Life Sciences) with an inter-assay coefficient of variation (CV) of $1.7 \%$.

Plasma insulin concentrations during the clamp and MTT were measured by ELISA (Millipore, Billerica, MA, USA) with inter-assay CV $<14 \%$ and intra-assay CV $<10 \%$. 
Plasma triglyceride (TG), non-esterified FA (NEFA), total cholesterol, and HDL-cholesterol concentrations were measured using commercially available kits for the ILab650 (Instrumentation Laboratory, Warrington, UK), with all inter-assay $\mathrm{CV}$ values being $<2.5 \%$ and all intraassay CV values being $<1.5 \%$.

Fasting tumor necrosis factor $\alpha(\mathrm{TNF} \alpha)$ and interleukin 6 (IL6) were measured by commercially available ELISA Kits (2B Scientific, Upper Heyford, UK) with inter-assay CV values $<4$ and $<18 \%$ respectively and intra-assay $\mathrm{CV}$ values $<7$ and $<10 \%$ respectively. Plasma adiponectin and leptin were measured using ELISA (Millipore) with intra-assay CV of 3.6 and $4.4 \%$ respectively.

Blood samples for C-peptide and total GLP1 analyses from the MTT were collected into potassium EDTA tubes containing 200 kallikrein inhibiting units (KIU) aprotinin $/ \mathrm{ml}$ of blood. Plasma samples were then measured by ELISA (Millipore), with inter- and intra-assay CV values $<25$ and $<6.5 \%$ for C-peptide and GLP1 respectively.

Serum short-chain FAs (SCFAs) were measured using a gas chromatography-based method as described previously $(15,16)$. For each sample, $1 \mu \mathrm{l}$ was injected into a Hewlett Packard 5890 Series II GC system fitted with a Nukol Capillary Column $(30 \mathrm{~m} \times 0.53 \mathrm{~mm} \times 1.0 \mu \mathrm{m}$, SUPELCO Analytical, Poole, Dorset, UK) and flame ionization detector. The peaks were integrated using Agilent ChemStation Software, and SCFA contents quantified by singlepoint internal standard method. Peak identity and internal response factors were determined using a $1 \mathrm{mM}$ calibration cocktail, including acetic, propionic, iso-butyric, butyric, iso-valeric, valeric, ethyl-butyric, and caproic acids.

\section{A priori and retrospective sample size}

Sample size was based on $n=15$ (7). Based on 15 participants completing this crossover study ( $\alpha=0.05$ ), there would be an $82 \%$ probability of detecting a treatment difference of $73.7 \mathrm{mmol} / \mathrm{l}$ per min in glucose area under the curve (AUC) 0-120 min, based on the assumption of a treatment S.D. of 84 , with postprandial glucose tolerance as the primary outcome measure. Both A-V uptake and MRI scanning were not performed due to problems with vascular access and unforeseen claustrophobia in a number of patients. Based on the actual participant numbers, the retrospective power estimate for detecting a difference in the A-V glucose uptake into muscle was $84 \%$, based on a treatment s.D. of 58 and a measured treatment effect of $111 \mathrm{mmol} / 1$ per $100 \mathrm{ml}$ tissue. For the MRI measures, the inter-individual variability found in this T2DM cohort was much higher than expected based on data available from those without diabetes. As such, this secondary outcome was significantly underpowered during the study, with only $21 \%$ power $(\alpha=0.05)$ due to a very high treatment variable s.D. of 4.2 .

\section{Calculations and statistical analyses}

LDL-cholesterol was calculated using the Friedewald equation (17). Fasted insulin sensitivity (\%S) and $\beta$-cell function $(\% \mathrm{~B})$ were assessed by the homeostatic model assessment (HOMA) (18), and postprandial insulin sensitivity was calculated using the Matsuda index (19).

During the clamp, EGP and glucose Rd were calculated using the model proposed by Steele (20) modified to include the use of stable isotopes. The volume of distribution was assumed to be $22 \%$ of body weight. The calculation was also modified for the inclusion of $\left[6,6-{ }^{2} \mathrm{H}_{2}\right]$ glucose in the dextrose infusion (21). Before calculation of glucose turnover, plasma glucose concentrations and glucose enrichment time courses were smoothed using optimal segments technique analysis (22). For each time point, EGP and Rd were calculated. Data are expressed as mean EGP and Rd from five sample values collected during each steady state (basal, low, and high dose).

AUC was calculated using the trapezoid rule for 0-120 min $\left(\mathrm{AUC}_{0-120 \mathrm{~min}}\right)$ for each of the postprandial metabolites. Incremental AUC (iAUC) was also calculated to allow for any significant differences in baseline concentrations. During the MTT, A-V differences in metabolite concentrations were calculated. Total FA uptake into muscle was calculated from the rate of TG and NEFA removal across the tissue as described previously (5).

All dietary analyses were carried out using nutritional analysis software (Dietplan6 Professional version, Forestfield Software, Horsham, UK) and average daily intake was calculated.

All statistical analyses were carried out using SPSS 19.0 for Windows. Statistical significance was taken as $P<0.05$. All data were normalized and analyzed using paired samples $t$-tests and time course data analyzed using repeated-measures ANOVA. Owing to the heterogeneity of the patient group, exploratory Pearson's correlation coefficients were computed to assess potential relationships. For these correlations, percentage change after consumption of HAM-RS2 compared with placebo was calculated. All results are expressed as mean \pm s.E.M.

\section{Results}

The inclusion of both supplements into habitual diets was well tolerated by the participants as assessed by the bowel

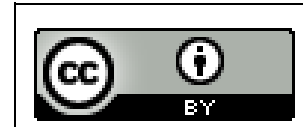

This work is licensed under a Creative Commons Attribution 3.0 Unported License. 
habit and symptom diaries. The only reported side effect was a mild but significant increase in the ratings of flatulence with HAM-RS2 compared with placebo $(1.1 \pm$ 0.2 vs $0.7 \pm 0.1$, respectively, $P=0.006$, measured on a scale of 0-4 (none to debilitating)).

There was no significant difference in energy intake between the HAM-RS2 and placebo $(2044 \pm 116$ vs $2089 \pm$ $119 \mathrm{kcal}$ respectively). There was also no significant difference in carbohydrate and fat intakes; however, protein intakes were significantly lower with the HAMRS2 compared with the placebo $(84.2 \pm 2.6$ vs $92.3 \pm 4.8 \mathrm{~g}$, $P=0.043)$. As would be expected, mean fiber intake increased significantly during the HAM-RS2 intervention $(60.1 \pm 1.3$ compared with $22.7 \pm 1.9 \mathrm{~g} ; P<0.001)$, which can be directly attributed to the HAM-RS2 supplement.

\section{Glucose metabolism and insulin sensitivity}

There was no significant difference, between the HAM-RS2 and the placebo at the end of the 12-week intervention, in fasting glucose or insulin (Table 1) and therefore no significant difference in fasting insulin sensitivity or $\beta$-cell function as assessed by HOMA (Table 1). Similarly, there was no significant difference between the interventions in long-term glucose control as assessed by HbA1c (Table 1).

During the two-step euglycemic-hyperinsulinemic clamp, no difference was observed between interventions in EGP at basal (placebo, $11.5 \pm 0.6 \mu \mathrm{mol} / \mathrm{kg}$ per min and HAM-RS2, $10.7 \pm 0.7 \mu \mathrm{mol} / \mathrm{kg}$ per min) or during the low dose insulin infusion (placebo, $4.6 \pm 0.3 \mu \mathrm{mol} / \mathrm{kg}$ per min and HAM-RS2, $5.0 \pm 0.4 \mu \mathrm{mol} / \mathrm{kg}$ per min). No significant differences were observed between interventions for glucose Rd at basal (placebo, $11.9 \pm 0.5 \mu \mathrm{mol} / \mathrm{kg}$ per min and HAM-RS2, $10.7 \pm 0.8 \mu \mathrm{mol} / \mathrm{kg}$ per min) or during the high dose insulin infusions (placebo, $47.0 \pm 5.4 \mu \mathrm{mol} / \mathrm{kg}$ per min and HAM-RS2, $47.3 \pm 6.0 \mu \mathrm{mol} / \mathrm{kg}$ per min). There were no differences in insulin concentrations throughout the clamp study. Postprandial glucose concentrations during the MTT exhibited a significant treatment by time interaction $(P=0.045$; Fig. $1 \mathrm{~A})$; this translated as a significant reduction in glucose $\mathrm{AUC}_{0-120}$ min with the HAM-RS2 $(P=0.036)$. The A-V sampling across the forearm muscle also showed a trend for higher glucose uptake with HAM-RS2 compared with the placebo ( $P=0.077$; Fig. $1 \mathrm{~B})$.

The lower postprandial glucose concentrations with HAM-RS2 were not associated with a significant lowering of the plasma insulin concentration; however, there was a significant time $\times$ treatment interaction effect on plasma C-peptide concentrations ( $P=0.038$; data not shown). There were no significant differences between the
Table 1 Anthropometric measurements, body fat depots, and fasting plasma concentrations taken after 12 weeks supplementation with $40 \mathrm{~g} /$ day HAM-RS2 compared with placebo. Mean \pm S.E.M. for 17 patients.

\begin{tabular}{|c|c|c|c|}
\hline & HAM-RS2 & Placebo & $P$ value \\
\hline Body weight $(\mathrm{kg})$ & $92.5 \pm 5.0$ & $91.7 \pm 5.1$ & NS \\
\hline BMI $\left(\mathrm{kg} / \mathrm{m}^{2}\right)$ & $31.0 \pm 1.3$ & $30.7 \pm 1.4$ & NS \\
\hline Fat mass $(\mathrm{kg})^{a}$ & $32.2 \pm 2.7$ & $31.8 \pm 2.9$ & NS \\
\hline Total AT $(I)^{\mathrm{b}}$ & $35.8 \pm 3.6$ & $34.5 \pm 4.0$ & NS \\
\hline Subcutaneous AT (I) & $26.2 \pm 2.9$ & $25.6 \pm 3.3$ & NS \\
\hline Internal AT (I) & $9.6 \pm 1.1$ & $9.0 \pm 1.0$ & NS \\
\hline $\mathrm{IHCL}^{\mathrm{b}}$ & $9.7 \pm 2.7$ & $10.0 \pm 3.3$ & NS \\
\hline Pancreas fat ${ }^{b}$ & $13.7 \pm 3.9$ & $10.5 \pm 3.2$ & NS \\
\hline S-IMCL ${ }^{\mathrm{b}}$ & $24.7 \pm 4.6$ & $19.3 \pm 3.7$ & 0.027 \\
\hline T-IMCL ${ }^{\mathrm{b}}$ & $7.9 \pm 1.1$ & $6.5 \pm 0.8$ & NS \\
\hline $\mathrm{HbA} 1 \mathrm{c}(\mathrm{mmol} / \mathrm{mol})$ & $46.8 \pm 1.5$ & $47.9 \pm 2.0$ & NS \\
\hline Glucose (mmol/l) & $6.2 \pm 0.2$ & $6.4 \pm 0.2$ & NS \\
\hline Insulin (pmol/l) & $49.1 \pm 7.4$ & $51.0 \pm 9.5$ & NS \\
\hline C-peptide (nmol/l) & $0.7 \pm 0.1$ & $0.7 \pm 0.1$ & NS \\
\hline HOMA \%S & $116.3 \pm 15.1$ & $115.9 \pm 13.9$ & NS \\
\hline HOMA \%B & $67.5 \pm 7.1$ & $63.5 \pm 6.8$ & NS \\
\hline NEFA $(\mu \mathrm{mol} / \mathrm{l})$ & $500 \pm 100$ & $600 \pm 50$ & 0.004 \\
\hline TG $(\mathrm{mmol} / \mathrm{l})$ & $1.4 \pm 0.1$ & $1.2 \pm 0.1$ & 0.039 \\
\hline $\begin{array}{l}\text { Total cholesterol } \\
(\mathrm{mmol} / \mathrm{l})\end{array}$ & $3.6 \pm 0.1$ & $3.4 \pm 0.2$ & NS \\
\hline $\begin{array}{l}\text { HDL-cholesterol } \\
(\mathrm{mmol} / \mathrm{l})\end{array}$ & $1.0 \pm 0.1$ & $1.0 \pm 0.1$ & NS \\
\hline $\begin{array}{l}\text { LDL-cholesterol } \\
(\mathrm{mmol} / \mathrm{l})\end{array}$ & $1.9 \pm 0.1$ & $1.8 \pm 0.1$ & NS \\
\hline GLP1 (pmol/l) & $11.4 \pm 1.9$ & $17.0 \pm 3.2$ & 0.049 \\
\hline Leptin (ng/ml) & $10.9 \pm 1.9$ & $10.2 \pm 1.8$ & NS \\
\hline Adiponectin (ng/ml) & $8232 \pm 1249$ & $7701 \pm 879$ & NS \\
\hline TNF $\alpha(p g / m l)$ & $5.1 \pm 2.6$ & $13.2 \pm 3.9$ & 0.013 \\
\hline IL6 (pg/ml) & $6.5 \pm 1.8$ & $3.8 \pm 1.5$ & NS \\
\hline Acetate $(\mu \mathrm{mol} / \mathrm{l})$ & $99.1 \pm 1.2$ & $98.3 \pm 6.1$ & NS \\
\hline Propionate $(\mu \mathrm{mol} / \mathrm{l})$ & $4.0 \pm 0.2$ & $7.5 \pm 1.4$ & 0.021 \\
\hline Butyrate $(\mu \mathrm{mol} / \mathrm{l})$ & $0.6 \pm 0.1$ & $1.1 \pm 0.1$ & $<0.001$ \\
\hline
\end{tabular}

AT, adipose tissue; IHCL, intrahepatocellular lipid; S-IMCL, soleus intramyocellular lipid; T-IMCL, tibialis IMCL; HOMA \%S, fasted oral insulin sensitivity assessed by homeostasis model assessment; HOMA \%B, $\beta$-cell function, assessed by HOMA (18).

a Measured by bioimpedance $(n=16)$.

body fat depots determined by MRS scanning $(n=14)$.

interventions for postprandial insulin sensitivity assessed by the Matsuda index (19).

\section{Fat metabolism}

Increased consumption of HAM-RS2 resulted in significantly higher fasting TG concentrations $(P=0.039$; Table 1) compared with the placebo, with no effect on cholesterol concentrations. After 12 weeks of increased HAM-RS2 consumption, fasting NEFA concentrations were significantly lower $(P=0.004$; Table 1$)$ and there was greater differential suppression of NEFA by insulin during the two-step clamp $(P=0.001 ;$ Fig. 2). During the MTT, there was no effect of treatment on postprandial

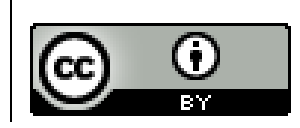

This work is licensed under a Creative Commons Attribution 3.0 Unported License. 

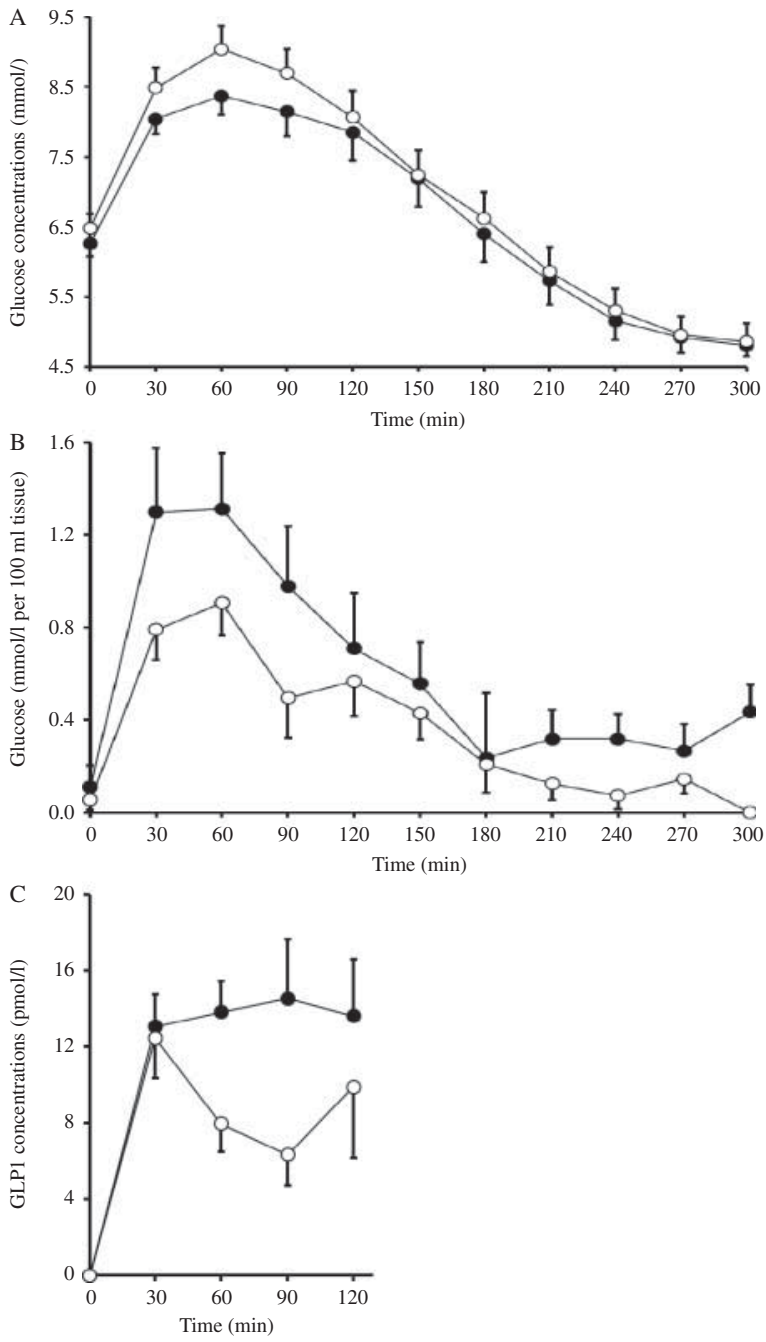

\section{Figure 1}

Postprandial glucose concentrations (A), glucose flux into the muscle tissue measured with arterio-venous sampling across the forearm muscle (B), and change from baseline postprandial GLP1 concentrations (C) during the MTT at the end of 12 weeks supplementation with HAM-RS2 (filled circle) compared with placebo (open circle). Mean + s.E.M.s for 16 patients ( $A$ and $C$ ) and 12 patients (B). There was a significant treatment $\times$ time interaction for the postprandial glucose concentrations as assessed by repeated measures ANOVA $(P=0.045)$, which corresponded to a significantly reduced $A \cup C_{0-120 \text { min }}$ following the HAM-RS2 supplement $(P=0.036)$ compared with paired $t$-test. Repeated measures ANOVA showed a trend for increased glucose uptake with the HAM-RS2 compared with the placebo $(P=0.077)$. There was a significantly greater GLP1 response with HAM-RS2 compared with the placebo $(P=0.009)$ compared with paired $t$-test on the $\mathrm{IAUC}_{0-120 \mathrm{~min}}$

concentrations of NEFA or TG and no effect on FA flux as measured by A-V sampling (data not shown); however, the percentage increase in FA uptake into muscle from placebo to HAM-RS2 intake correlated significantly both with an increase in glucose disposal (Rd) measured during the clamp study $(P=0.036)$ and with glucose flux measured by A-V sampling in the postprandial state $(P=0.004)$.

\section{Inflammatory markers, SCFA, and hormones}

Increased intake of HAM-RS2 for 12 weeks resulted in significantly lower fasting TNF $\alpha$ concentrations $(P=0.013$; Table 1) but had no effects on fasting IL6 concentrations (Table 1). There was also no effect of HAM-RS2 treatment on either fasting plasma leptin or adiponectin concentrations (Table 1). Fasting plasma propionate and butyrate concentrations were significantly lower $(P=0.021$ and $P<0.001$ respectively) following the HAM-RS2 compared with the placebo, but there were no significant differences between treatments for plasma acetate concentrations (Table 1).

Fasting GLP1 concentrations were significantly lower $(P=0.049)$ following HAM-RS2 compared with placebo; however, there was a significantly greater meal GLP1 excursion with HAM-RS2 than with the placebo $(P=0.009$; Fig. 1C).

\section{Vascular function and blood pressure}

There were no significant differences in blood pressure, carotid-femoral pulse wave velocity or any of the clinical markers of vascular function (data not shown) following HAM-RS2.

\section{Body weight and composition}

Despite no significant differences in body weight, BMI, or fat mass (Table 1), following 12 weeks of increased HAMRS2 intake, soleus intramyocellular lipid (S-IMCL) content was significantly higher compared with placebo $(P=0.027$; Table 1); the tibialis IMCL (T-IMCL) was also higher following HAM-RS2 although not significantly (Table 1). This increase in S-IMCL was significantly correlated with the reduction in both fasting NEFA $(P=0.022)$ and HbA1c $(P=0.017)$. There were no other significant differences between interventions for liver, pancreatic or AT fat depots, assessed by MRS scanning.

\section{Discussion}

Although consumption of HAM-RS2 has been extensively investigated in healthy groups and those with the metabolic syndrome $(3,4,5,6,7,10)$, this work represents the first attempt at translation of these findings into an efficacious dietary treatment for human T2DM. In individuals with well-controlled diabetes (mean HbA1c,

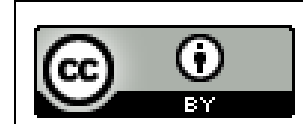




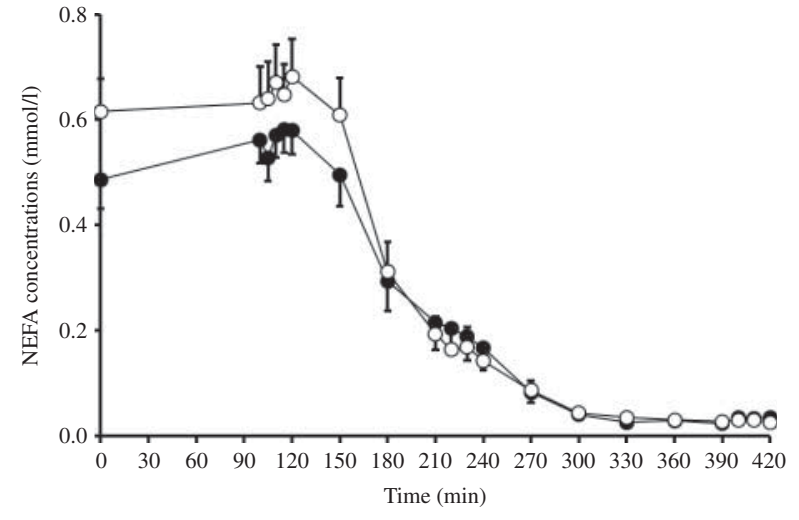

\section{Figure 2}

Plasma NEFA concentrations during the two-step euglycemic-hyperinsulinemic clamp at the end of 12 weeks supplementation with HAM-RS2 (filled circle) compared with placebo (open circle). Mean + s.E.M.s for 15 patients. Repeated measures ANOVA showed greater differential suppression of NEFA by insulin with the HAM-RS2 compared with the placebo $(P=0.001)$.

$46.6 \mathrm{mmol} / \mathrm{mol}$ and target UK level for T2DM, $48 \mathrm{mmol} / \mathrm{mol}$ ), taking oral hypoglycemics, HAM-RS2 intake resulted in a significant improvement in the meal glucose handling (Fig. 1) without a change in medication, habitual diet, exercise, or indeed weight loss. There was, however, no change in the HbA1c following supplementation, but as the intervention was relatively short at 12 weeks in patients already at target HbA1c levels, a longer period may be required for this to become evident.

Although a primary aim of this work was to translate positive results from individuals at increased risk of diabetes into those with the condition $(3,7)$, it is evident that there are clear differences in the responsiveness between the patient groups to the dietary change. Unexpectedly, intravenous insulin sensitivity as assessed by the hyperinsulinemic clamp technique was not affected by HAM-RS2 intake, despite a positive improvement in oral glucose handling. This disparity between i.v. and oral glucose disposal might imply a gut-mediated factor to be responsible for the effects, a phenomenon often attributed to GLP1. Indeed, GLP1, a well-defined incretin, was found to be elevated postprandially after HAM-RS2 intake, again a finding which was not found in our previous published work in those without diabetes (23) but has been reported in studies of RS in animal models (24). Interestingly, there was no effect of this elevated GLP1 on postprandial insulin levels and so any effect on postprandial glucose disposal may have been through insulin-independent mechanisms. GLP1 has been shown to directly increase muscle glucose uptake in rodent models (25), with the GLP1 receptor recently localized to human skeletal muscle (26). GLP1 acutely raises nitric oxide (NO) levels and so acute changes in both microvascular recruitment (27) and endothelial function (28) at the level of the muscle are believed to be involved in this effect. In the current study, glucose uptake across forearm muscle measured directly using A-V sampling was increased following HAM-RS2 intake and against a background of elevated GLP1 (Fig. 1) although failing to reach statistical significance $(P=0.077)$ in the 12 patients in whom arterialized blood samples were obtained. Interestingly, on comparing data obtained from those with T2DM with results obtained using an identical technique in metabolic syndrome patients (7), it appeared that HAM-RS2 intake improved muscle glucose uptake in T2DM patients to levels found in pre-diabetes/metabolic syndrome patients. As HAM-RS2 intake in metabolic syndrome has been shown to normalize glucose uptake to 'healthy' levels, it could be hypothesized that a longer period of supplementation would have the potential to improve meal glucose tolerance further in T2DM than achieved during this initial 12 -week investigation.

Other metabolic effects attributed to HAM-RS2 intake, such as an improved anti-lipolytic activity of insulin in AT lipolysis, have now also been confirmed in the present study. Both at fasting and under insulin stimulation during the clamp, NEFA concentration was significantly decreased, an effect previously attributed to both stimulation of AT FFA2/3 receptors directly by products of microbial fermentation (29) and to changes at the transcriptional level (7). However, it should be noted that HAM-RS2 consumption did not increase fasting serum SCFA in this study; indeed butyrate and propionate levels were significantly lowered. This may be counterintuitive, but earlier work using the same supplement in healthy individuals also found no impact on fasting SCFA concentrations (7) and, indeed, does not discount a change in fecal/luminal concentrations, which may be involved in the increased stimulation of GLP1 through the FFAR 2/3 receptor. Indeed, recent work using microbial transfer has demonstrated that increasing butyrate-producing bacterial species, for example, does not necessarily result in an increase in butyrate production, implying that the impact of both microbes and dietary fibers is more complex than that of simple SCFA levels (30). An explanation for the lower level of propionate could be the increased clearance into peripheral tissue as demonstrated previously (5) although has yet to be demonstrated in vivo in diabetes. Although historically the focus of the reduction in NEFA with fiber feeding has always been in relation to AT content, paradoxically perhaps, a significant increase in S-IMCL

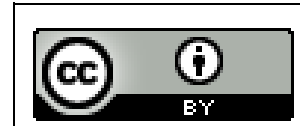

This work is licensed under a Creative Commons Attribution 3.0 Unported License. 
was found despite an improvement in glucose tolerance. We have also previously shown this in people with the metabolic syndrome in whom there was a substantial nonsignificant increase (50\% increase) in S-IMCL (3). Ectopic fat storage per se does not cause insulin resistance (31) and it is hypothesized that the increased partitioning of FAs toward TG storage in a neutral-lipid droplet may be beneficial in blunting the lipotoxicity of lipid species such as ceramides, diacylglycerol (DAG), and fatty acyl coA (32). Indeed, both a single-exercise session (33) and prolonged fasting (34) have been shown to partition more FA toward TG synthesis in skeletal muscle, whereas weight-loss alone does not (35). In this study, the reduction in fasting plasma NEFA correlated significantly with the increase in IMCL in soleus muscle $(P=0.022)$. Although the peripheral rate of glucose disposal from the clamp study (Rd) was not significantly different with HAM-RS2 intake, the change in Rd correlated significantly with the change in FA flux into forearm muscle. Combining these lines of evidence suggests that skeletal muscle is a major metabolic target for HAM-RS2 in T2DM, as was found previously in metabolic syndrome. It would be interesting to speculate whether the molecular changes within muscle tissue parallel those found with exercise training such as improved mitochondrial content/function (36).

In accordance with previous data from our group $(5,7)$, the metabolic impact of HAM-RS2 intake in diabetes would appear to be confined to the periphery (AT and muscle). A new finding in this group of individuals with diabetes was a reduction in obesity-associated inflammation independent from any changes in body fat volume per se. Again, as in our previous work in metabolic syndrome $(3,7)$, our current study found no evidence for an effect on hepatic TG storage or the ability of insulin to inhibit hepatic glucose output (EGP). However, it should be noted that not all the effects observed on lipids could be considered beneficial/neutral. A significant increase in plasma TG was observed in these individuals with T2DM, nonsignificant increases in TG have been observed by our group previously, when RS is used in large doses. In the current study, the increase although statistically significant is unlikely to be clinically significant as participants had TG levels within the reference range. However, the implication for individuals with baseline hyperlipidemia is unknown and warrants investigation.

In conclusion, this is the first RS feeding study in human T2DM where the metabolic effects of RS (rather than a manipulation of dietary glycemic index/glycemic load (37)) have been investigated. HAM-RS2 intake improved meal glucose tolerance in patients with existing good diabetic-control due to a mechanism which appears to involve increased muscle uptake of FAs and increased S-IMCL. However, as a caveat, changes in both ectopic TG distribution and plasma TG were found, the clinical significance of which is unknown. Further work is now warranted to elucidate the molecular mechanisms within muscle tissue attributable to HAM-RS2, which would be vital in terms of recommending diet/exercise interventions to maximize the benefits for muscle glucose uptake. A larger scale intervention should now be undertaken in patients using high-fiber foods, with less well-controlled diabetes and over a longer time frame before a change to the evidenced-based dietary guidelines could be proposed.

\section{Declaration of interest}

There were no conflicts of interest relevant to this paper. The supplements were supplied free of charge from Ingredion, Inc. (Bridgewater, NJ, USA) but there was no industrial involvement in the design of the study or the interpretation of the data.

\section{Funding}

The study was funded by the Diabetes UK Alec and Beryl Warren Award (BDA 09/0003895).

\section{Author contribution statement}

C L Bodinham conducted the clinical experiments, analyzed the data, and wrote the manuscript; L Smith assisted with the conduction of clinical experiments, analysis of data, and edited the manuscript; E L Thomas acquired and analyzed the data and edited the manuscript, J D Bell edited the manuscript; J R Swann acquired and analyzed the data and edited the manuscript; A Costabile acquired and analyzed the data; D Russell-Jones supervised the clinical work; A M Umpleby analyzed the data and edited the manuscript; M D Robertson assisted with the conduction of clinical experiments, analyzed the data, and wrote the manuscript. M D Robertson is the guarantor of the work and had full access to all of the data in the study and takes responsibility for the integrity of the data and the accuracy of the data analysis. Parts of this study were presented in abstract form at the 73rd Scientific Sessions of the American Diabetes Association, Chicago, June 2013.

\section{Acknowledgements}

The authors thank Nicola Jackson, Joanne Batt, and Francesca Robertson for laboratory assistance and John Wright for medical assistance. The research team acknowledges the support of the National Institute for Health Research, through the Primary Care Research Network.

\section{References}

1 Post RE, Mainous AG III, King DE \& Simpson KN. Dietary fiber for the treatment of type 2 diabetes mellitus: a meta-analysis. Journal of the American Board of Family Medicine 201225 16-23. (doi:10.3122/ jabfm.2012.01.110148)

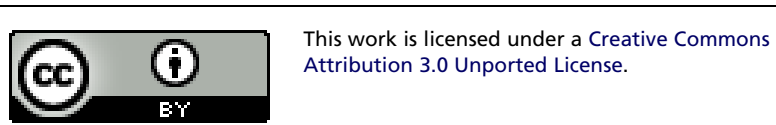


2 SACN. Scientific Committee on Nutrition Position statement on dietary fibre. 2008. (available at: http://www.sacn.gov.uk/reports_position_statements/position_statements/draft_sacn_position_statement_on_dietary_ fibre_health_and_the_dietary_fibre_definition_-_august_2008.html)

3 Johnston KL, Thomas EL, Bell JD, Frost GS \& Robertson MD. Resistant starch improves insulin sensitivity in metabolic syndrome. Diabetic Medicine 201027 391-397. (doi:10.1111/j.1464-5491.2010.02923.x)

4 Maki KC, Pelkman CL, Finocchiaro ET, Kelley KM, Lawless AL, Schild AL \& Rains TM. Resistant starch from high-amylose maize increases insulin sensitivity in overweight and obese men. Journal of Nutrition 2012142 717-723. (doi:10.3945/jn.111.152975)

5 Robertson MD, Bickerton AS, Dennis AL, Vidal H \& Frayn KN. Insulinsensitizing effects of dietary resistant starch and effects on skeletal muscle and adipose tissue metabolism. American Journal of Clinical Nutrition 200582 559-567.

6 Robertson MD, Currie JM, Morgan LM, Jewell DP \& Frayn KN. Prior short-term consumption of resistant starch enhances postprandial insulin sensitivity in healthy subjects. Diabetologia 200346 659-665.

7 Robertson MD, Wright JW, Loizon E, Debard C, Vidal H, ShojaeeMoradie F, Russell-Jones D \& Umpleby AM. Insulin-sensitizing effects on muscle and adipose tissue after dietary fiber intake in men and women with metabolic syndrome. Journal of Clinical Endocrinology and Metabolism 201297 3326-3332. (doi:10.1210/jc.2012-1513)

8 Keenan MJ, Zhou J, McCutcheon KL, Raggio AM, Bateman HG, Todd E, Jones CK, Tulley RT, Melton S, Martin RJ et al. Effects of resistant starch, a non-digestible fermentable fiber, on reducing body fat. Obesity 2006 14 1523-1534. (doi:10.1038/oby.2006.176)

9 Zhou J, Martin RJ, Tulley RT, Raggio AM, McCutcheon KL, Shen L, Danna SC, Tripathy S, Hegsted M \& Keenan MJ. Dietary resistant starch upregulates total GLP-1 and PYY in a sustained day-long manner through fermentation in rodents. American Journal of Physiology. Endocrinology and Metabolism 2008295 E1160-E1166. (doi:10.1152/ ajpendo.90637.2008)

10 Bodinham CL, Smith L, Wright J, Frost GS \& Robertson MD. Dietary fibre improves first-phase insulin secretion in overweight individuals PLoS ONE 20127 e40834. (doi:10.1371/journal.pone.0040834)

11 Robertson MD. Dietary-resistant starch and glucose metabolism. Current Opinion in Clinical Nutrition and Metabolic Care 201215 362-367. (doi:10.1097/MCO.0b013e3283536931)

12 Bantle JP, Wylie-Rosett J, Albright AL, Apovian CM, Clark NG, Franz MJ, Hoogwerf BJ, Lichtenstein AH, Mayer-Davis E, Mooradian AD et al. Nutrition recommendations and interventions for diabetes: a position statement of the American Diabetes Association. Diabetes Care 200831 (Suppl 1) S61-S78.

13 Mann JI, De Leeuw I, Hermansen K, Karamanos B, Karlstrom B, Katsilambros N, Riccardi G, Rivellese AA, Rizkalla S, Slama G et al. Evidence-based nutritional approaches to the treatment and prevention of diabetes mellitus. Nutrition, Metabolism, and Cardiovascular Diseases 200414 373-394. (doi:10.1016/S0939-4753(04)80028-0)

14 Shojaee-Moradie F, Jackson NC, Jones RH, Mallet AI, Hovorka R \& Umpleby AM. Quantitative measurement of 3-O-methyl-D-glucose by gas chromatography-mass spectrometry as a measure of glucose transport in vivo. Journal of Mass Spectrometry 199631 961-966. (doi:10 1002/(SICI)1096-9888(199609)31:9 < 961::AID-JMS359>3.0.CO;2-H)

15 Fernandes J, Vogt J \& Wolever TM. Insulin increases short-term markers for colonic fermentation similarly in healthy and hyperinsulinaemic humans. European Journal of Clinical Nutrition 201165 1279-1286. (doi:10.1038/ejcn.2011.116)

16 Vogt JA, Pencharz PB \& Wolever TM. L-Rhamnose increases serum propionate in humans. American Journal of Clinical Nutrition 2004 80 89-94.

17 Friedewald WT, Levy RI \& Fredrickson DS. Estimation of the concentration of low-density lipoprotein cholesterol in plasma, without use of the preparative ultracentrifuge. Clinical Chemistry 1972 18 499-502.

18 Matthews DR, Hosker JP, Rudenski AS, Naylor BA, Treacher DF \& Turner RC. Homeostasis model assessment: insulin resistance and $\beta$-cell function from fasting plasma glucose and insulin concentrations in man. Diabetologia 198528 412-419. (doi:10.1007/BF00280883)

19 Matsuda $\mathrm{M} \&$ DeFronzo RA. Insulin sensitivity indices obtained from oral glucose tolerance testing: comparison with the euglycemic insulin clamp. Diabetes Care 199922 1462-1470. (doi:10.2337/diacare.22.9.1462)

20 Steele R. Influences of glucose loading and of injected insulin on hepatic glucose output. Annals of the New York Academy of Sciences 1959 82 420-430. (doi:10.1111/j.1749-6632.1959.tb44923.x)

21 Finegood DT, Bergman RN \& Vranic M. Estimation of endogenous glucose production during hyperinsulinemic-euglycemic glucose clamps. Comparison of unlabeled and labeled exogenous glucose infusates. Diabetes 198736 914-924. (doi:10.2337/diab.36.8.914)

22 Finegood DT \& Bergman RN. Optimal segments: a method for smoothing tracer data to calculate metabolic fluxes. American Journal of Physiology 1983244 E472-E479.

23 Bodinham CL, Al-Mana NM, Smith L \& Robertson MD. Endogenous plasma glucagon-like peptide-1 following acute dietary fibre consumption. British Journal of Nutrition 2013110 1429-1433.

24 Shen L, Keenan MJ, Raggio A, Williams C \& Martin RJ. Dietary-resistant starch improves maternal glycemic control in Goto-Kakizaki rat. Molecular Nutrition \& Food Research 201155 1499-1508. (doi:10.1002/ mnfr.201000605)

25 Ayala JE, Bracy DP, James FD, Julien BM, Wasserman DH \& Drucker DJ. The glucagon-like peptide-1 receptor regulates endogenous glucose production and muscle glucose uptake independent of its incretin action. Endocrinology 2009150 1155-1164. (doi:10.1210/en.2008-0945)

26 Green CJ, Henriksen TI, Pedersen BK \& Solomon TP. Glucagon like peptide-1-induced glucose metabolism in differentiated human muscle satellite cells is attenuated by hyperglycemia. PLoS ONE 20127 e44284. (doi:10.1371/journal.pone.0044284)

27 Chai W, Dong Z, Wang N, Wang W, Tao L, Cao W \& Liu Z. Glucagonlike peptide 1 recruits microvasculature and increases glucose use in muscle via a nitric oxide-dependent mechanism. Diabetes 201261 888-896. (doi:10.2337/db11-1073)

28 Nystrom T, Gutniak MK, Zhang Q, Zhang F, Holst JJ, Ahren B \& Sjoholm A. Effects of glucagon-like peptide-1 on endothelial function in type 2 diabetes patients with stable coronary artery disease. American Journal of Physiology. Endocrinology and Metabolism 2004287 E1209-E1215. (doi:10.1152/ajpendo.00237.2004)

29 Ge H, Li X, Weiszmann J, Wang P, Baribault H, Chen JL, Tian H \& Li Y. Activation of $G$ protein-coupled receptor 43 in adipocytes leads to inhibition of lipolysis and suppression of plasma free fatty acids. Endocrinology 2008149 4519-4526. (doi:10.1210/en.2008-0059)

30 Vrieze A, Van Nood E, Holleman F, Salojarvi J, Kootte RS, Bartelsman JF, Dallinga-Thie GM, Ackermans MT, Serlie MJ, Oozeer R et al. Transfer of intestinal microbiota from lean donors increases insulin sensitivity in individuals with metabolic syndrome. Gastroenterology 2012143 913-916 (e917). (doi:10.1053/j.gastro.2012.06.031)

31 Kotronen A, Seppala-Lindroos A, Bergholm R \& Yki-Jarvinen H. Tissue specificity of insulin resistance in humans: fat in the liver rather than muscle is associated with features of the metabolic syndrome. Diabetologia 200851 130-138. (doi:10.1007/s00125-007-0867-x)

32 Timmers S, de Vogel-van den Bosch J, Hesselink MK, van Beurden D, Schaart G, Ferraz MJ, Losen M, Martinez-Martinez P, De Baets $\mathrm{MH}$ Aerts JM et al. Paradoxical increase in TAG and DAG content parallel the insulin sensitizing effect of unilateral DGAT1 overexpression in rat skeletal muscle. PLOS ONE 20116 e14503. (doi:10.1371/journal.pone. 0014503)

33 Schenk S \& Horowitz JF. Acute exercise increases triglyceride synthesis in skeletal muscle and prevents fatty acid-induced insulin resistance. Journal of Clinical Investigation 2007117 1690-1698. (doi:10.1172/ JCI30566)

34 Stannard SR, Thompson MW, Fairbairn K, Huard B, Sachinwalla T \& Thompson $\mathrm{CH}$. Fasting for $72 \mathrm{~h}$ increases intramyocellular lipid content in nondiabetic, physically fit men. American Journal of Physiology. Endocrinology and Metabolism 2002283 E1185-E1191.

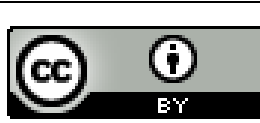


35 Toledo FG, Menshikova EV, Azuma K, Radikova Z, Kelley CA, Ritov VB \& Kelley DE. Mitochondrial capacity in skeletal muscle is not stimulated by weight loss despite increases in insulin action and decreases in intramyocellular lipid content. Diabetes 200857 987-994. (doi:10.2337/db07-1429)

36 Meex RC, Schrauwen-Hinderling VB, Moonen-Kornips E, Schaart G, Mensink M, Phielix E, van de Weijer T, Sels JP, Schrauwen P \& Hesselink MK. Restoration of muscle mitochondrial function and metabolic flexibility in type 2 diabetes by exercise training is paralleled by increased myocellular fat storage and improved insulin sensitivity. Diabetes 201059 572-579. (doi:10.2337/ db09-1322)

37 Kwak JH, Paik JK, Kim HI, Kim OY, Shin DY, Kim HJ \& Lee JH. Dietary treatment with rice containing resistant starch improves markers of endothelial function with reduction of postprandial blood glucose and oxidative stress in patients with prediabetes or newly diagnosed type 2 diabetes. Atherosclerosis 2012224 457-464. (doi:10.1016/j.atherosclerosis.2012.08.003)

Received in final form 11 March 2014

Accepted 26 March 2014
This work is licensed under a Creative Commons Attribution 3.0 Unported License. 